\title{
Macroalgas de ambientes lóticos da região centro-oeste do Paraná, região sul do Brasil
}

\author{
Ciro Cesar Zanini Branco ${ }^{1,2}$, Cleto Kaveski Peres ${ }^{1}$, \\ Rogério Antônio Krupek ${ }^{I}$ \& Fernando Rodrigo Bertusso ${ }^{I}$ \\ ${ }^{1}$ Departamento de Ciências Biológicas, Laboratório de Biologia Aquática, \\ Faculdade de Ciências e Letras, Universidade Estadual Paulista - UNESP, \\ Av. Dom Antônio, 2100, CEP 19806-900, Assis, SP, Brasil \\ ${ }^{2}$ Autor para correspondência: czbranco@assis.unesp.br
}

BRANCO, C.C.Z., PERES, C.K., KRUPEK, R.A. \& BERTUSSO, F.R. Stream macroalgae from the midwestern region of Paraná State, southern Brazil. Biota Neotrop., 9(2): http://www.biotaneotropica.org.br/ v9n2/en/abstract?inventory+bn01909022009.

\begin{abstract}
Taxonomic studies focusing on the inventory of lotic macroalgae in different regions are important contributions to the basic knowledge for these algal communities. In this context, the main objective of this investigation was to accomplish a floristic survey of the stream macroalgal communities in the mid-west region of Paraná State, Southern Brazil, a poorly investigated subtropical area. Twenty-two stream segments were sampled by means of cross-transect technique. Twenty-four macroalgal taxa were found, including 18 subgeneric taxa and 6 vegetative groups. Chlorophyta was the most species rich division (13 taxa $=54 \%$ of the total), followed by Cyanophyta (five taxa $=21 \%$ ) and Rhodophyta and Heterokontophyta $(3$ taxa $=12.5 \%)$. Phormidium retzii (C. Agardh) Gomont (Cyanophyta) was the most widespread species, and mats the predominant morphological type. Although all the macroalgal taxa had been previously described from Brazilian streams, 5 species are new records from Paraná State. Even with no additional species reported, the survey in the mid-west region of Paraná State are relevant, since it expanded the geographical and environmental distribution of the stream macroalgal communities in Brazil.
\end{abstract}

Keywords: floristic survey, stream macroalgae, subtropical region, Chlorophyta, Paraná State.

BRANCO, C.C.Z., PERES, C.K., KRUPEK, R.A. \& BERTUSSO, F.R. Macroalgas de ambientes lóticos da região Centro-Oeste do Paraná, região Sul do Brasil. Biota Neotrop., 9(2): http://www.biotaneotropica.org. br/v9n2/pt/abstract?inventory+bn01909022009.

Resumo: Estudos taxonômicos enfocando o inventário de macroalgas de ambientes lóticos em diferentes regiões são considerados importantes contribuições para o conhecimento básico destas comunidades algais. Neste contexto, o principal objetivo desta investigação foi realizar um levantamento florístico das comunidades de macroalgas da região centro-oeste do estado do Paraná, região Sul do Brasil, uma área de clima subtropical pouco investigada. Vinte e dois segmentos de rios/riachos foram amostrados através da técnica da transeção. Vinte e quatro táxons de macroalgas foram encontrados, incluindo 18 táxons subgenéricos e 6 grupos vegetativos. Chlorophyta foi a divisão com maior número de espécies (13 táxons $=54 \%$ ), seguida por Cyanophyta (cinco táxons $=21 \%$ ) e Rhodophyta e Heterokontophyta (3 táxons $=12,5 \%$ cada uma). Phormidium retzii (C. Agardh) Gomont (Cyanophyta) foi a espécie melhor distribuída, e emaranhados de filamentos o tipo morfológico predominante. Apesar de todos os táxons já tenham sido previamente descritos para ambientes lóticos brasileiros, 5 espécies são novos registros para o estado do Paraná. Embora sem nenhuma espécie nova registrada, o levantamento taxonômico da região centro-oeste do estado do Paraná é extremamente relevante, uma vez que ele amplia as distribuições geográfica e ambiental das comunidades de macroalgas no Brasil.

Palavras-chave: levantamento florístico, macroalgas lótica, região subtropical, Chlorophyta, Estado do Paraná. 


\section{Introdução}

O componente macroscópico das algas bentônicas de ambientes lóticos ["macroalgas" como proposto por Sheath \& Cole (1992)] tem despertado muito interesse, tanto sobre os seus aspectos taxonômicos quanto ecológicos, já que estes organismos têm sido reconhecidos como importantes na produção primária desses ecossistemas (Necchi et al. 1994, Branco \& Pereira 2002, Lowe \& LaLiberte 2006). Por conta deste interesse, vários estudos foram desenvolvidos, principalmente nas últimas décadas, em diversas partes do mundo (América do Norte - Sheath \& Burkholder 1985, Sheath et al. 1986, Sheath \& Cole 1992, Verb \& Vis 2001; Europa - Kawecka 1980, 1982, Johansson 1982, John \& Moore 1985; Australásia - Biggs \& Price 1987, Entwisle 1990, Filkin et al. 2003).

No Brasil, merecem ser citados diversos estudos que enfocaram estes organismos (Necchi et al. 1995, 1997, 2000, 2003, Branco \& Necchi 1996, 1997, 1998, Krupek et al. 2008, Branco et al. 2008), entretanto, levantamentos florísticos ainda são relativamente escassos para esses grupos de organismos. Até o momento, os estudos mais relevantes envolvendo as macroalgas lóticas foram desenvolvidos nas regiões tropicais do Estado de São Paulo. Alguns exemplos neste contexto são os trabalhos de Branco \& Necchi (1996), para riachos da região leste da Mata Atlântica (encosta atlântica) e Necchi et al. (1997), para a região noroeste. Exceções são os recentes levantamentos florísticos desenvolvidos no Estado do Paraná: Branco et al. (2008) estudaram riachos de uma unidade de conservação da floresta ombrófila mista (Mata de Araucária); Peres et al. (2008), amostraram uma região de floresta ombrófila densa (Mata Atlântica) da região litorânea do estado; Krupek et al. (2008), realizaram levantamento em uma bacia de drenagem da região central do Estado.

Considerando que o Brasil é um país de dimensões continentais com uma grande variedade de regiões tropicais e subtropicais nas quais as condições climáticas mudam enormemente, mais levantamentos taxonômicos de outras regiões do país são necessários para produzir novas e importantes informações sobre a flora e distribuição ecológica das comunidades de macroalgas lóticas. Neste contexto insere-se o presente estudo, com amostragens conduzidas na região centro-oeste do Estado do Paraná $\left(23^{\circ} 58^{\prime}-26^{\circ} 13^{\prime} \mathrm{S}\right.$ e $50^{\circ} 53^{\prime}$ $\left.54^{\circ} 35^{\prime} \mathrm{W}\right)$, Sul do Brasil. O objetivo foi ampliar e aprimorar as informações taxonômicas sobre estes grupos algais na região subtropical do país, uma área praticamente desconhecida quanto às comunidades de macroalgas lóticas. Informações sobre a distribuição ecológica das comunidades de macroalgas desta mesma região geográfica foram apresentadas em um trabalho separado (Branco et al. 2009).

\section{Material e Métodos}

A região de estudos está contida no Terceiro Planalto Paranaense, que compreende as terras situadas a oeste da escarpa da Esperança (Figura 1). Geologicamente corresponde ao vasto derrame de rochas eruptivas (basalto, diabásios e meláfiros) e aos depósitos de arenito (Botucatu e Caiuá) da era Mesozóica (Wons 1982). Mais especificamente, a região de estudos localiza-se na porção do Terceiro Planalto, chamada de Planalto de Guarapuava, delimitada pelos rios Piquiri (ao norte), Iguaçu (ao sul) e Paraná (a oeste). O relevo da região representa um plano de declive que forma a encosta da escarpa da Esperança (Maack 1981), sendo este um dos seus acidentes mais notáveis. As altitudes variam de $1.250 \mathrm{~m}$ na escarpa, declinando em direção oeste, atingindo valores inferiores a $550 \mathrm{~m}$ (Wons 1982). A região amostrada apresenta três tipos vegetacionais principais, segundo Wons (1982): a) Campos limpos - predomínio de gramíneas que geralmente refletem solos mais pobres; apresentam-se entremeados com matas ciliares e capões isolados; b) Matas de Araucária - compreende a mata subtropical de coníferas, onde o Pinheiro do Paraná [Araucaria angustifolia (Bert.) O. Ktze.] aparece como principal elemento paisagístico; e c) Mata Pluvial Subtropical - diferencia-se da Mata de Araucária por ocupar terras inferiores a $500 \mathrm{~m}$ de altitude e pela ausência dos pinheiros. Os tipos climáticos principais, segundo a classificação de Köeppen, apresentaram-se como variações do clima subtropical úmido (Wons 1982).

O programa de amostragem foi conduzido em 22 segmentos de rios/riachos (Figura 1) visitados uma vez durante o período compreendido entre o final do outono ao começo da primavera no ano de 2002 (24 de maio a 25 de outubro de 2002). Este período é descrito como
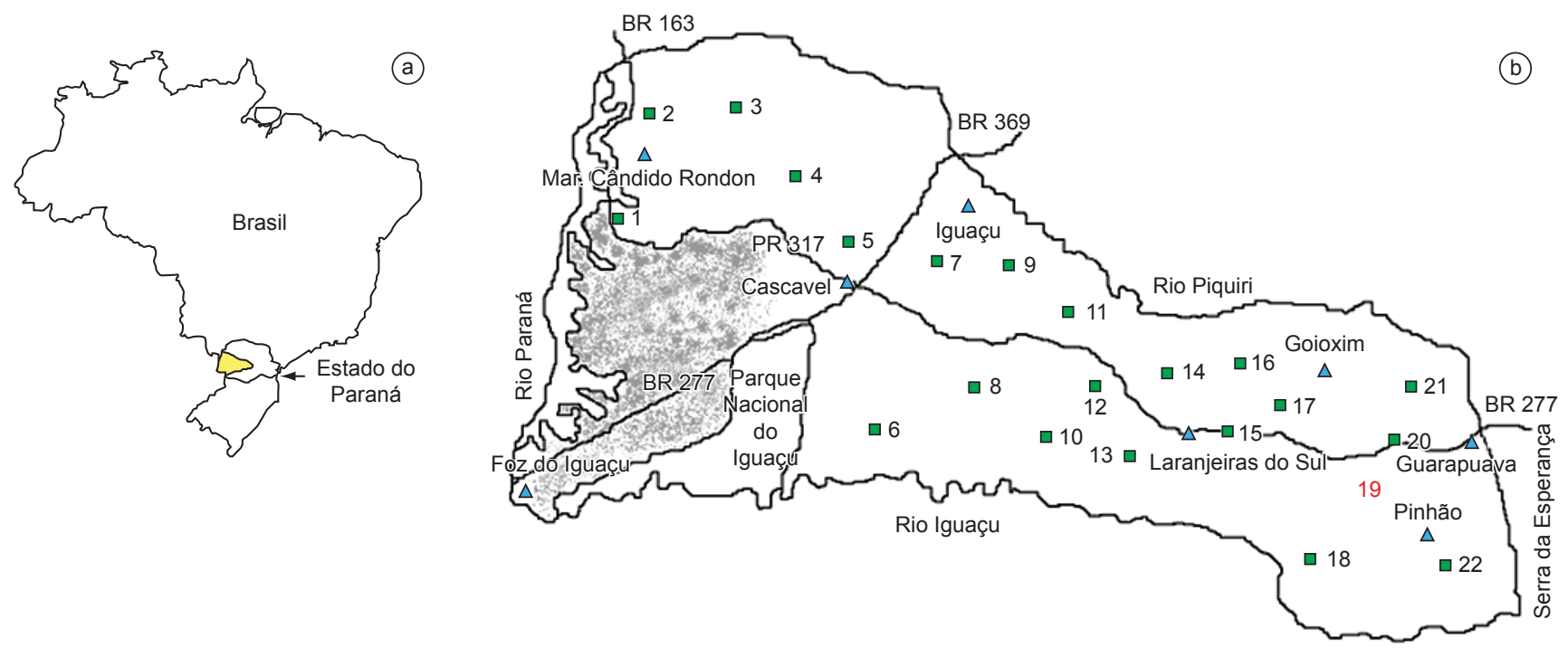

Figura 1. a) Localização da região centro-oeste do estado do Paraná; e b) Detalhe da região centro-oeste do Paraná mostrando os pontos de amostragem (quadrados) e as principais cidades (triângulos).

Figure 1. a) Localization of the mid-west region of Paraná State; and b) Detail of the mid-west region of Paraná State showing the sampling sites (squares) and main cities (triangles). 
sendo o de maior diversidade e abundância de espécies de macroalgas para rios/riachos do estado de São Paulo (Necchi \& Pascoaloto 1993) e foi tomado como base pela ausência de informações para outras regiões brasileiras.

Os pontos de amostragem consistiram de trechos de 10 metros de comprimento, que foram totalmente examinados quanto a presença das espécies que compunham as comunidades de macroalgas. Os rios/ riachos variaram quanto ao tamanho, sendo amostrados desde os de $1^{\text {a }}$ ordem até os de $5^{\mathrm{a}}$ ordem. Os tipos morfológicos de macroalgas foram definidos de acordo com Sheath \& Cole (1992).

As amostras de algas foram preservadas imediatamente após a coleta em formaldeído tamponado 4\% (Johansson 1982). Em laboratório o material preservado foi observado em microscópio LEICA, mod. DM-1000 equipado com uma câmera digital LEICA, mod. DFC-280. As medidas microscópicas e fotomicrografias foram realizadas com o auxílio do software LEICA IM-50 Image Manager. Os exemplares foram identificados, sempre que possível, até o nível de espécie e a classificação dos táxons dentro das divisões, classes e ordens seguiu o sistema proposto por Hoek et al. (1995), exceto para Cyanophyta, onde foi adotado o sistema de Anagnostidis \& Komarek (1988, 1990) e Komárek \& Anagnostidis (1989). Para cada táxon infragenérico foram fornecidas as seguintes informações: a) breve descrição do material contendo as informações taxonômicas completas, desde que o material ainda não tivesse sido descrito em trabalhos prévios no estado do Paraná, ou quando mesmo que previamente descritos fossem identificadas variações morfométricas significativas; b) distribuição na área de estudos, com a identificação do ponto de ocorrência e c) características ambientais (valores máximos, mínimos e médios) medidas nos rios/riachos onde o táxon foi registrado. Comentários taxonômicos foram incorporados sempre que problemas de identificação ou diferenças taxonômicas importantes foram registrados.

Para a identificação das características ambientais de cada rio/ riacho investigado, e conseqüentemente da amplitude ecológica da ocorrência das macroalgas identificadas na área de estudos, as seguintes variáveis ambientais foram medidas em cada ponto de amostragem: temperatura da água, condutividade específica, velocidade da correnteza, $\mathrm{pH}$ e oxigênio dissolvido. Todas as variáveis foram medidas diretamente em campo com o auxílio de um analisador de água multiparâmetro da marca Consort, modelo C535. Ainda, foi medida a velocidade da correnteza a uma profundidade de $5 \mathrm{~cm}$ em três pontos eqüidistantes dentro do intervalo amostrado com um fluxômetro mecânico General Oceanics 2030R.

\section{Resultados}

\section{DIVISÃO CYANOPHYTA}

\section{CLASSE CYANOPHYCEAE}

\section{ORDEM OSCILLATORIALES}

1. Geitlerinema splendidum (Gomont) Anagnostidis (Figura 2) Pl. Syst. Evol. 164: 33-46, 1989.

As características do material concordam com a descrição da espécie encontrada por Branco et al. (2008) em riachos da Floresta Nacional de Irati.

Distribuição e característica dos riachos $(\mathrm{N}=1)$ : ponto 21 ; temperatura $18,3{ }^{\circ} \mathrm{C}$, condutividade específica $9,5 \mu \mathrm{S} . \mathrm{cm}^{-1}, \mathrm{pH} 6,7$, saturação de oxigênio $63 \%$, velocidade da correnteza $38 \mathrm{~cm} / \mathrm{s}$.

Comentários: Esta espécie tem sido descrita para ambientes lênticos ou solos úmidos do Brasil (Sant'Anna \& Azevedo 1995); entretanto, Branco et al. (1999) a reportaram para ambientes lóticos do estado de São Paulo, exatamente o mesmo ambiente onde a espécie foi registrada durante o presente estudo.

2. Microcoleus subtorulosus Gomont (Figura 3) Ann. Sci. Nat. Bot., Ser. 7, 15: 360, 1892.

As características do material concordam com a descrição da espécie encontrada por Branco et al. (2008) em riachos da Floresta Nacional de Irati.

Distribuição e característica dos riachos $(\mathrm{N}=3)$ : pontos 3,6 , 17; temperatura $16,3-19,1^{\circ} \mathrm{C}(\bar{\chi}=18,1)$, condutividade específica 24,8-65,6 $\mu{\mathrm{S} . \mathrm{cm}^{-1}}^{-1}(\bar{\chi}=36,4), \mathrm{pH} 7,6-8,3(\bar{\chi}=7,9)$, saturação de oxigênio $53-55 \%(\bar{\chi}=54)$, velocidade da correnteza $13-84 \mathrm{~cm} / \mathrm{s}$ $(\bar{\chi}=59)$.

3. Phormidium retzii (C.Agardh) Gomont (Figura 4) Ann Sci. Nat. Bot., Sér. 7, 16: 175, 1892.

As características do material concordam com a descrição da espécie encontrada por Branco et al. (2008) em riachos da Floresta Nacional de Irati.

Distribuição e característica dos riachos $(\mathrm{N}=7)$ : pontos 2, 4, 6 , $8,9,14,16$; temperatura $16,3-22,7^{\circ} \mathrm{C}(\bar{\chi}=18,8)$, condutividade específica 7,5-65,6 $\mu{\mathrm{S} . \mathrm{cm}^{-1}}^{(}(\bar{\chi}=32,5), \mathrm{pH}$ 5,7-7,6 $(\bar{\chi}=6,7)$, saturação de oxigênio $47-68 \%(\bar{\chi}=59)$, velocidade da correnteza $42-91 \mathrm{~cm} / \mathrm{s}(\bar{\chi}=68)$.

\section{ORDEM NOSTOCALES}

1. Scytonema arcangeli Bornet ex Flahault (Figura 5) Ann. Sci. Nat. Bot., Sér. 7, 5: 92, 1886.

Talo formado por tufos verde-azulados; filamentos regularmente ramificados, 15,0-30,0 $\mu \mathrm{m}$ diâmetro, ramificações falsas duplas; bainha hialina a marrom-amarelada, fina, algumas vezes com lamelação paralela e margens onduladas; tricomas constritos ou não, 6,6-15,8 $\mu \mathrm{m}$ diâmetro, 4,0-15,0 $\mu \mathrm{m}$ comprimento; compr./diâm. 0,3-1,5; conteúdo celular granulado, verde-azulado; heterocitos raros, quadráticos ou cilíndricos, 6,5-12,0 $\mu \mathrm{m}$ comprimento, 8,3-15,7 $\mu \mathrm{m}$ diâmetro; hormogônios presentes.

Distribuição e característica dos riachos $(\mathrm{N}=1)$ : ponto 22; temperatura $18,4{ }^{\circ} \mathrm{C}$, condutividade específica $10,8 \mu \mathrm{S} . \mathrm{cm}^{-1}$, pH 6,19, saturação de oxigênio $65 \%$, velocidade da correnteza $99 \mathrm{~cm} / \mathrm{s}$.

Comentários: Esta espécie já havia sido reportada para riachos paranaenses (Peres et al. 2008), porém, o material analisado apresentou dimensões celulares com maiores amplitudes do que na descrição anterior. Entretanto, os valores métricos registrados para a região centro-oeste encontram-se perfeitamente de acordo com aqueles reportados para a espécie em outros estudos realizados em riachos brasileiros (p. ex., Branco et al. 1999).

2. Tolypothrix distorta Bornet et Flahault (Figura 6) Ann. Sci. Nat. Bot., Sér. 7, 5: 119, 1887.

Talo formado por tufos verde-azulados, filamentos regularmente ramificados, 9,5-15,3 $\mu \mathrm{m}$ diâmetro; ramificações falsas simples; bainha hialina, fina, homogênea; tricomas constritos nos septos, 7,5-11,3(-14,0) $\mu \mathrm{m}$ diâmetro, 2,5-4,0 $\mu \mathrm{m}$ comprimento; compr./diâm. 0,2-0,6; conteúdo celular granulado, heterocitos arredondados ou cônico-arredondados, 10,5-13,0 $\mu \mathrm{m}$ comprimento, 12,0-13,7 $\mu \mathrm{m}$ diâmetro.

Distribuição e característica dos riachos $(\mathrm{N}=2)$ : pontos 10 , 13; temperatura $20,4-21,3{ }^{\circ} \mathrm{C}(\bar{\chi}=20,8)$, condutividade específica $63,8-73,4 \mu \mathrm{S} . \mathrm{cm}^{-1}(\bar{\chi}=68,6)$, pH 7,21-7,45 $(\bar{\chi}=7,33)$, saturação de oxigênio $74-76 \%(\bar{\chi}=75)$, velocidade da correnteza $21-34 \mathrm{~cm} / \mathrm{s}(\bar{\chi}=27.5)$. 

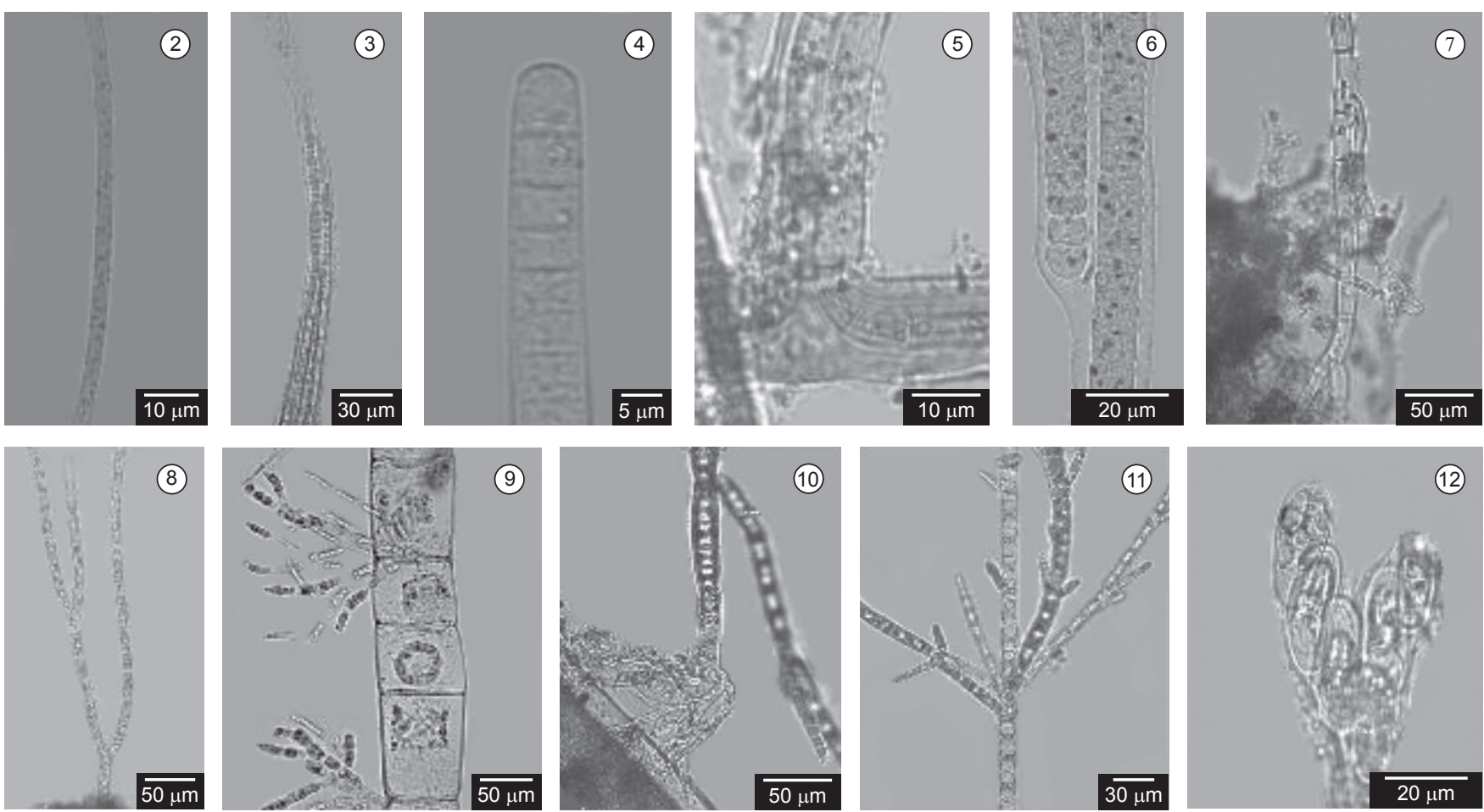

Figuras 2-12. 2) Geitlerinema splendidum; 3) Microcoleus subtorulosus; 4) Phormidium retzii; 5) Scytonema arcangeli; 6) Tolypothrix distorta; 7-8) Chaetophora pisiformis, 7) rizóides, 8) sistema ereto; 9) Draparnaldia mutabilis; 10-11) Stigeoclonium fasciculare, 10) rizóides, 11) sistema ereto; 12) Ecballocystis pulvinata.

Figures 2-12. 2) Geitlerinema splendidum; 3) Microcoleus subtorulosus; 4) Phormidium retzii; 5) Scytonema arcangeli; 6) Tolypothrix distorta; 7-8) Chaetophora pisiformis, 7) rhizoids, 8) erect system; 9) Draparnaldia mutabilis; 10-11) Stigeoclonium fasciculare, 10) rhizoids, 11) erect system; 12) Ecballocystis pulvinata.

Comentários: Os espécimes observados apresentaram tricomas menores do que o geralmente reportado na literatura; entretanto, todas as outras características estão de acordo com a circunscrição da espécie (Branco et al. 1999). Por isso, acreditamos que a variação encontrada representa apenas ampliação na variabilidade morfométrica daquele caráter da espécie. Este é o primeiro registro desta espécie para o Estado do Paraná.

\section{DIVISÃO CHLOROPHYTA}

\section{CLASSE CHLOROPHYCEAE}

\section{ORDEM CHAETOPHORALES}

1. Chaetophora pisiformis (Roth) C. Agardh (Figuras 7-8) Dispositio Algarum Sueicae 4; 43, 1812.

Planta com matriz gelatinosa macia, verde claro; filamentos eretos di- ou tricotomicamente ramificados; células do eixo principal cilíndricas, raramente infladas, 23,4-41,6 $\mu \mathrm{m}$ comprimento, 7,8-11,7 $\mu$ m diâmetro, compr./diâm. 3,0-5,2; sistema basal composto exclusivamente por rizóides surgindo das células iniciais do filamento ereto, ocasionalmente a partir de células da região mediana do filamento.

Distribuição e característica dos riachos $(\mathrm{N}=1)$ : ponto 19 ; temperatura $18,3{ }^{\circ} \mathrm{C}$, condutividade específica $10,2 \mu \mathrm{S} . \mathrm{cm}^{-1}$, $\mathrm{pH} 6,27$, saturação de oxigênio $53 \%$, velocidade da correnteza $42 \mathrm{~cm} / \mathrm{s}$.

Comentários: Este é o primeiro registro desta espécie para o Estado do Paraná.
2. Draparnaldia mutabilis (Roth) Bory (Figura 9) Ann. Mus. National d'Hist. Natur. 12: 405, 1808.

Planta consistindo de filamentos ramificados, envolvido por uma mucilagem macia, quase fluida; eixo principal distinto com células cilíndricas infladas, (56,0-)78,0-146,0 $\mu \mathrm{m}$ comprimento, 44,0-102,0 $\mu \mathrm{m}$ diâmetro, compr./diâm. 0,9-1,9; fascículos laterais quase sempre sem raque distinta, ovalados; ramificações di- ou tricotômicas, alternas ou opostas; células dos ramos laterais cilíndricas, 25,0-50,0 $\mu \mathrm{m}$ comprimento, 8,0-12,0 $\mu \mathrm{m}$ diâmetro, compr./diâm. 2,1-5,4; sistema basal formado por massa profusa de rizóides ramificados.

Distribuição e característica dos riachos $(\mathrm{N}=1)$ : ponto 7; temperatura $22,3{ }^{\circ} \mathrm{C}$, condutividade específica $60,2 \mu \mathrm{S} . \mathrm{cm}^{-1}, \mathrm{pH} 7,22$, saturação de oxigênio $93 \%$, velocidade da correnteza $56 \mathrm{~cm} / \mathrm{s}$.

Comentários: Esta espécie já foi descrita para riachos paranaenses (Branco et al. 2008), porém, o material analisado apresentou maiores amplitudes das dimensões celulares do que na descrição anterior.

3. Stigeoclonium fasciculare Kützing (Figuras 10-11) Bot. Ztg. 5: 177,1847

Planta verde claro; sistema basal composto por rizóides partindo de uma massa de células esféricas; sistema ereto bem desenvolvido e ramificado; ramificações alternas ou opostas, frequentemente formada por fascículos nas proximidades da região apical; células do eixo principal cilíndricas, similar em formato e tamanho ao longo da planta, 25,0-40,0 $\mu \mathrm{m}$ comprimento, 11,5-17,0 بm diâmetro, compr./diâm. 2,4-6,2; células dos ramos 
laterais cilíndricas ou levemente infladas, 7,4-20,0 $\mu \mathrm{m}$ long, 6,5-13,8 m diâmetro, compr./diâm. 1,1-1,7; ápices dos ramos acuminados, formação de zoósporos evidenciada pela presença de filamentos multisseriados.

Distribuição e característica dos riachos $(\mathrm{N}=1)$ : ponto 7; temperatura $22,3{ }^{\circ} \mathrm{C}$, condutividade específica $60,2 \mu \mathrm{S} . \mathrm{cm}^{-1}, \mathrm{pH} 7,22$, saturação de oxigênio $93 \%$, velocidade da correnteza $56 \mathrm{~cm} / \mathrm{s}$.

Comentários: Este é o primeiro registro desta espécie para o Estado do Paraná.

\section{ORDEM CHLOROCCOCALES}

1. Ecballocystis pulvinata Bohlin var. pulvinata (Figura 12) Sv. Vet.Akad. Hand., 23(7): 1, 1897

As características do material concordam com a descrição da espécie encontrada por Peres et al. (2008) em riachos da Serra da Prata.

Distribuição e característica dos riachos $(\mathrm{N}=1)$ : ponto 18 ; temperatura $16,4^{\circ} \mathrm{C}$, condutividade específica $15,5 \mu \mathrm{S} . \mathrm{cm}^{-1}, \mathrm{pH} 6,76$, saturação de oxigênio $50 \%$, velocidade da correnteza $75 \mathrm{~cm} / \mathrm{s}$.

\section{ORDEM DESMIDIALES}

1. Desmidium grevillii (Kützing ex Rafs) De Bary (Figura 13) Conjugate 42: pl. 4, 1858

Filamentos enrolados, envolvidos por bainha de mucilagem; células aproximadamente mais longas do que largas, levemente constritas, 62,5-75,0 $\mu \mathrm{m}$ comprimento, 20,0-25,0 $\mu \mathrm{m}$ diâmetro; vista vertical elíptica.
Distribuição e característica dos riachos $(\mathrm{N}=1)$ : ponto 21 ; temperatura $18,3{ }^{\circ} \mathrm{C}$, condutividade especifica $9,5 \mu \mathrm{S} . \mathrm{cm}^{-1}, \mathrm{pH} 6,7$, saturação de oxigênio $63 \%$, velocidade da correnteza $38 \mathrm{~cm} / \mathrm{s}$.

\section{ORDEM OEDOGONIALES}

\section{Bulbochaete sp. (Figura 14)}

Filamentos ramificados com células sub-cilíndricas, 15,0-20,0 $\mu \mathrm{m}$ diâmetro, 25,0-32,5 $\mu \mathrm{m}$ comprimento. Oogônios e anterídios não observados.

Distribuição e característica dos riachos $(\mathrm{N}=1)$ : ponto 19 ; temperatura $18,3{ }^{\circ} \mathrm{C}$, condutividade específica $10,2 \mu \mathrm{S} . \mathrm{cm}^{-1}$, pH 6,27, saturação de oxigênio 53\%, velocidade da correnteza $42 \mathrm{~cm} / \mathrm{s}$.

Comentários: Somente uma única população estéril de Bulbochaete foi encontrada na área de estudos, tornando impossível a sua identificação em nível específico.

\section{Oedogonium sp. (Figura 15)}

Filamentos com células cilíndricas, (10,5-)12,5-23,4 $\mu \mathrm{m}$ diâmetro, 22,5-32,5(-55,9) um comprimento. Oogônios e anterídios não observados.

Distribuição e característica dos riachos $(\mathrm{N}=4)$ : pontos 9, $13,17,19$; temperatura $18,3-21,3{ }^{\circ} \mathrm{C}(\bar{\chi}=20,2)$, condutividade específica 10,2-63,8 $\mu \mathrm{S} . \mathrm{cm}^{-1}(\bar{\chi}=36,6), \mathrm{pH} 6,27-7,45(\bar{\chi}=6,83)$, saturação de oxigênio $53-76 \%(\bar{\chi}=66.7)$, velocidade da correnteza $34-91 \mathrm{~cm} / \mathrm{s}(\bar{\chi}=55.7)$.

Comentários: Somente populações estéreis de Oedogonium foram encontradas na área de estudos, tornando impossível a identificação em nível específico.
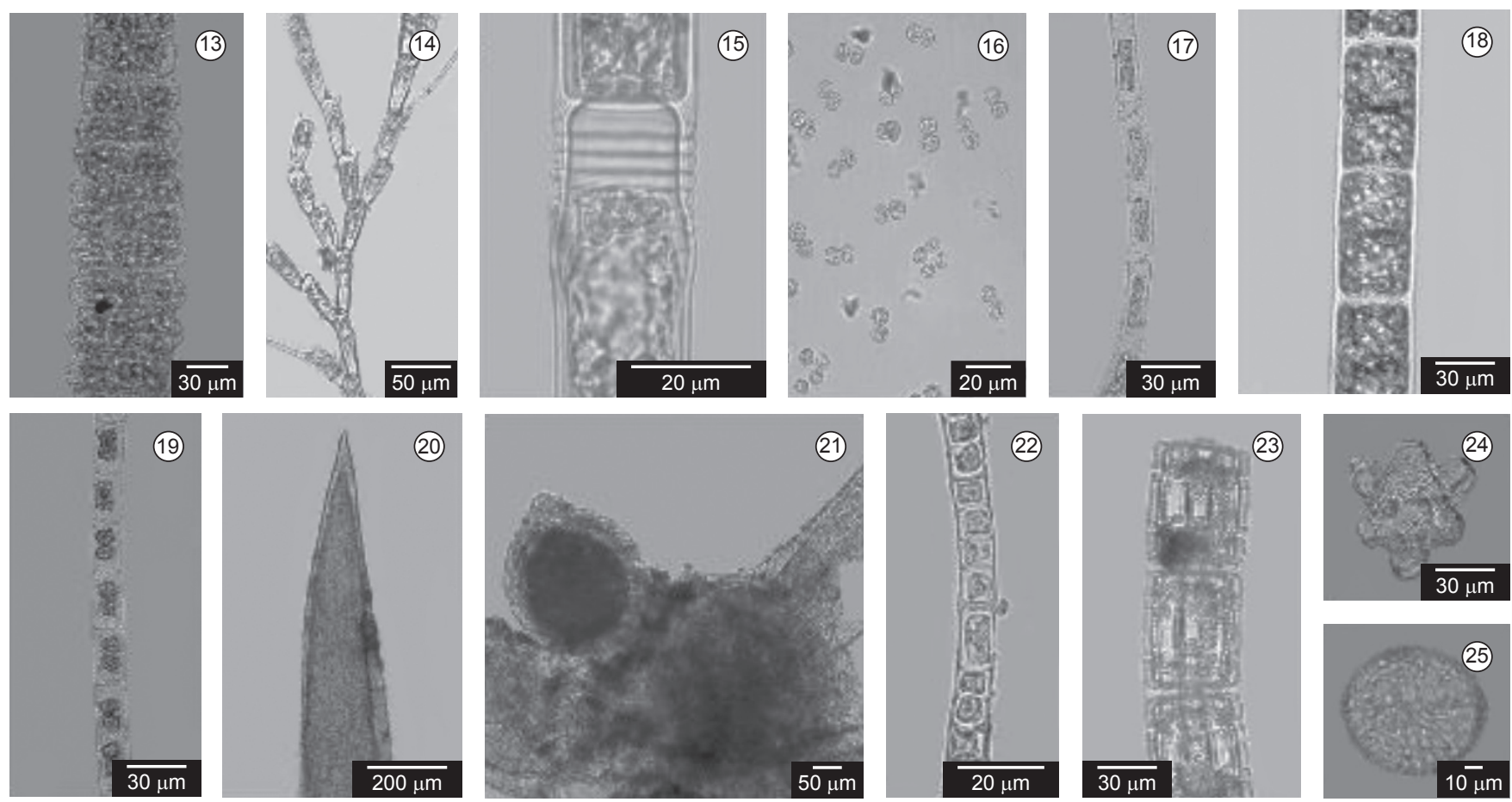

Figuras 13-25. 13) Desmidium grevillii; 14) Bulbochaete sp.; 15) Oedogonium sp.; 16) Tetraspora lubrica; 17) Mougeotia sp.; 18) Spirogyra sp.; 19) Zygnema sp.; 20-21) Nitella subglomerata, 20) detalhe do dáctilo, 21) oogônio (núcula); 22) Klebsormidium fluitans; 23-24) Hydrosera whampöensis, 23) colônia filamentosa, 24) vista valvar; 25) Pleurosira laevis.

Figures 13-25. 13) Desmidium grevillii; 14) Bulbochaete sp.; 15) Oedogonium sp.; 16) Tetraspora lubrica; 17) Mougeotia sp.; 18) Spirogyra sp.; 19) Zygnema sp.; 20-21) Nitella subglomerata, 20) detail of dactyls, 21) oogonium (nucule); 22) Klebsormidium fluitans; 23-24) Hydrosera whampöensis, 23) filamentous colony, 24) valvar view; 25) Pleurosira laevis. 


\section{ORDEM TETRASPORALES}

1. Tetraspora lubrica (Roth) C. Agardh (Figura 16) Syst. Algar., 1824

As características do material concordam com a descrição da espécie encontrada por Krupek et al. (2008) em riachos da bacia do rio das Pedras, Guarapuava.

Distribuição e características do riacho $(\mathrm{N}=2)$ : pontos 19,21 ; temperatura $18,3{ }^{\circ} \mathrm{C}(\bar{\chi}=18,3)$, condutividade específica 9,5$10,2 \mu{\mathrm{S} . \mathrm{cm}^{-1}}^{-}(\bar{\chi}=9,8), \mathrm{pH} 6,2-6,6(\bar{\chi}=6,4)$, saturação de oxigênio $53-63 \%(\bar{\chi}=58)$, velocidade da correnteza $38-42 \mathrm{~cm} / \mathrm{s}(\bar{\chi}=40)$.

\section{ORDEM ZYGNEMATALES}

\section{Mougeotia sp. (Figura 17)}

Filamentos formando emaranhados, células cilíndricas, 10,4-32,5 $\mu \mathrm{m}$ de diâmetro, 37,7-132,5 $\mu \mathrm{m}$ de comprimento. Zigósporos não observados.

Distribuição e características dos riachos $(\mathrm{N}=1)$ : pontos 2 ; temperatura $19,8{ }^{\circ} \mathrm{C}$, condutividade específica $6,2 \mu \mathrm{S} . \mathrm{cm}^{-1}$, $\mathrm{pH} 6,6$, saturação de oxigênio $53 \%$, velocidade da correnteza $81 \mathrm{~cm} / \mathrm{s}$.

Comentários: Somente uma única população estéril de Mougeotia foi encontrada na área de estudos, tornando impossível a sua identificação em nível específico.

\section{Spirogyra sp. (Figura 18)}

Filamentos formando emaranhados; células cilíndricas, 26,5-74,2 $\mu \mathrm{m}$ de diâmetro, 57,5-180,2 $\mu \mathrm{m}$ de comprimento. Zigósporos não observados.

Distribuição e características dos riachos $(\mathrm{N}=4)$ : pontos 13 , 16, 17, 21; temperatura $18,3-21,3{ }^{\circ} \mathrm{C}(\bar{\chi}=19)$, condutividade específica 9,5-63,8 $\left.\mu{\mathrm{S} . \mathrm{cm}^{-1}}^{(\bar{\chi}}=40,4\right), \mathrm{pH} 6,4-7,4(\bar{\chi}=6,8)$, saturação de oxigênio $55-74 \%(\bar{\chi}=64)$, velocidade da correnteza $7-60 \mathrm{~cm} / \mathrm{s}(\bar{\chi}=30)$.

Comentários: Somente populações estéreis de Spirogyra foram encontradas na área de estudos, tornando impossível a identificação em nível específico.

\section{Zygnema sp. (Figura 19)}

Filamentos formando emaranhados, células cilíndricas, 14,3-35,0 $\mu \mathrm{m}$ de diâmetro, 13-52,5 $\mu \mathrm{m}$ de comprimento; cloroplastos estrelados, 2 por célula, presença de um pirenóide central por célula. Zigósporos não observados.

Distribuição e características do riacho $(\mathrm{N}=2)$ : pontos 2,21 , 22 ; temperatura $17,2-18,4{ }^{\circ} \mathrm{C}(\bar{\chi}=18)$, condutividade específica $6,2-10,8 \mu \mathrm{S}_{\mathrm{cm}}{ }^{-1}(\bar{\chi}=8,83), \mathrm{pH} 6,2-6,7(\bar{\chi}=6,5)$, saturação de oxigênio $53-64 \%(\bar{\chi}=60)$, velocidade da correnteza $38-99 \mathrm{~m} / \mathrm{s}$ $(\bar{\chi}=73)$.

Comentários: Somente populações estéreis de Zygnema foram encontradas na área de estudos, tornando impossível a identificação em nível específico.

\section{CLASSE CHAROPHYCEAE}

\section{ORDEM CHARALES}

1. Nitella subglomerata A. Braum (Figs. 20-21) Mber. Dt. Akad. Wiss. Berlin, 1858: 356. 1859.

Plantas monóicas, 10-25 cm de altura, sem incrustação calcária; verticilos estéreis bem desenvolvidos, verticilos férteis reduzidos, formando capítulos; capítulos numerosos, distribuídos por toda a planta, 1-3 por verticilos, semi-esférico a cônico, frouxos, 2-6 mm diâmento, gametângios solitários ou em conjunto; núculas 1-5 por nós, sésseis, 220-600 $\mu \mathrm{m}$ comprimento, 160-380 $\mu \mathrm{m}$ diâmetro, 7-8 convoluções, corônula 19,6-55,3 ㅆm comprimento, 28,5-65,2 $\mu \mathrm{m}$ diâmetro.

Distribuição e característica dos riachos $(\mathrm{N}=4)$ : pontos 9 , $13,17,19$; temperatura $18,0-18,3{ }^{\circ} \mathrm{C}(\bar{\chi}=18,3)$, condutividade específica $10,2-43,7 \mu \mathrm{S} . \mathrm{cm}^{-1}(\bar{\chi}=26,9)$, pH 6,2, saturação de oxigênio $53-66,5 \%(\bar{\chi}=59,7)$, velocidade da correnteza $42-43 \mathrm{~cm} / \mathrm{s}$ $(\bar{\chi}=42,5)$.

\section{ORDEM KLEBSORMIDIALES}

\section{Klebsormidium fluitans (Gay) Lokhorst (Figura 22)}

Plantas formadas por filamentos emaranhados; filamentos usualmente enrolados, às vezes retos, com articulações em forma de joelho; células 3,9-9,1 $\mu \mathrm{m}$ de diâmetro, 5,2-10,4 $\mu \mathrm{m}$ de comprimento, compr./diâm. 0,6-1,4 $\mu \mathrm{m}$, cilíndricas ou doliformes; paredes celulares finas, usualmente com peças em $\mathrm{H}$ na região dos septos; cloroplastos parietais, ocupando a maior parte da circunferência celular.

Distribuição e características do riacho $(\mathrm{N}=1)$ : ponto 20 ; temperatura $18,0{ }^{\circ} \mathrm{C}$, condutividade específica $43,7 \mu \mathrm{S} . \mathrm{cm}^{-1}$, pH 6,2, saturação de oxigênio $66,5 \%$, velocidade da correnteza $43 \mathrm{~cm} / \mathrm{s}$.

Comentários: Este é o primeiro registro desta espécie para o estado do Paraná.

\section{DIVISÃO HETEROKONTOPHYTA}

\section{CLASSE BACILLARIOPHYCEAE}

\section{ORDEM CENTRALES}

1. Hydrosera whampoensis (Schwartz) Deby (Figs. 23-24) J. Microgr. 15: 209, 1891.

As características do material concordam com a descrição da espécie encontrada por Landucci \& Ludwig (2005).

Distribuição e características do riacho $(\mathrm{N}=1)$ : ponto 11 ; temperatura $18,2^{\circ} \mathrm{C}$, condutividade específica $51,7 \mu \mathrm{S} . \mathrm{cm}^{-1}, \mathrm{pH} 7,1$, saturação de oxigênio $69 \%$, velocidade da correnteza $16 \mathrm{~cm} / \mathrm{s}$.

\section{ORDEM TRICERATIALES}

1. Pleurosira laevis (Ehrenberg) Compère (Figura 25) Bacillaria 5: 117-178, 1982, Figuras 1-17, 20, 39.

Colônia formada por pseudofilamentos densamente emaranhados, células com valvas circulares a subcirculares, 50-64 $\mu \mathrm{m}$ diâmetro, 14-16 estrias em $10 \mu \mathrm{m}$; superfície valvar reta; estrias multisseriadas e radiadas desde o centro valvar e descendo pelo manto; grânulos podem estar dispersos pela superfície valvar.

Distribuição e características dos riachos $(\mathrm{N}=2)$ : pontos 5, 11; temperatura $16,6-18.2{ }^{\circ} \mathrm{C}(\bar{\chi}=17,4)$, condutividade específica 51,7-135,4 $\mu \mathrm{S} . \mathrm{cm}^{-1}(\bar{\chi}=93,5), \mathrm{pH} 7.1-7,9(\bar{\chi}=7,5)$, saturação de oxigênio $67-69 \%(\bar{\chi}=68)$, velocidade da correnteza $16-77 \mathrm{~cm} / \mathrm{s}$ $(\bar{\chi}=46,5)$.

\section{CLASSE XANTOPHYCEAE}

\section{ORDEM VAUCHERIALES}

\section{Vaucheria sp. (Figura 26)}

Talo formado por densos emaranhados de filamentos sifonáceos; sifões estéreis, escassamente ramificados; 5.5-87.5 $\mu \mathrm{m}$ de diâmetro. Oogônios e anterídios não observados.

Distribuição e características do riacho $(\mathrm{N}=4)$ : pontos 7 , $10,11,16$; temperatura $17,9-22,3{ }^{\circ} \mathrm{C}(\bar{\chi}=19,4)$, condutividade específica $27,1-73,4 \mu \mathrm{S} . \mathrm{cm}^{-1}(\bar{\chi}=51,1), \mathrm{pH} 5,3-7,2(\bar{\chi}=6,7)$, 
saturação de oxigênio 48-93\% ( $\bar{\chi}=70)$, velocidade da correnteza $16-89 \mathrm{~cm} / \mathrm{s}(\bar{\chi}=48)$.

Comentários: Somente espécimes estéreis foram encontrados, assim sendo, não foi possível a identificação em nível específico.

\section{DIVISÃO RHODOPHYTA}

\section{CLASSE FLORIDEOPHYCEAE}

\section{ORDEM BATRACHOSPERMALES}

1. Batrachospermum vogesiacum Schultz ex Skuja (Figuras 27-28) Arch. Hydrobiol. Suppl. 15: 623. 1938.

As características do material concordam com a descrição da espécie encontrada por Krupek et al. (2008) em riachos da bacia do rio das Pedras, Guarapuava.

Distribuição e características dos riachos $(\mathrm{N}=2)$ : pontos 15 , 18 ; temperatura $16,4-20,5^{\circ} \mathrm{C}(\bar{\chi}=18,5)$, condutividade específica
$15,5-58,4 \mu \mathrm{S} . \mathrm{cm}^{-1}(\bar{\chi}=37)$, pH 6,8-6,9 $(\bar{\chi}=6,85)$, saturação de oxigênio $50-65 \%(\bar{\chi}=57)$, velocidade da correnteza $20-75 \mathrm{~cm} / \mathrm{s}$ $(\bar{\chi}=47)$.

2. Batrachospermum puiggarianum Grunow in Wittrock et Nordstedt (Figure 29) Alg. Aq. Dul. Exsic. 11: 1, 1883.

As características do material concordam com a descrição da espécie encontrada por Krupek et al. (2008) em riachos da bacia do rio da Pedras, Guarapuava.

Distribuição e característica dos riachos $(\mathrm{N}=1)$ : ponto 7; temperatura $22,3{ }^{\circ} \mathrm{C}$, condutividade específica $60,2 \mu \mathrm{S} . \mathrm{cm}^{-1}, \mathrm{pH} 7,22$, saturação de oxigênio $93 \%$, velocidade da correnteza $56 \mathrm{~cm} / \mathrm{s}$.

Comentários: Além da presença do gametófito desta espécie, também foi registrada a ocorrência do estágio "Chantransia".

3. Sirodotia delicatula Skuja (Figuras 30-31) Arch. Hydrobiol. Suppl. 15: 614. 1938.

Planta monóica ou dióica, moderada ou abundantemente mucilaginosa, ramificação irregular, abundante, eixo principal
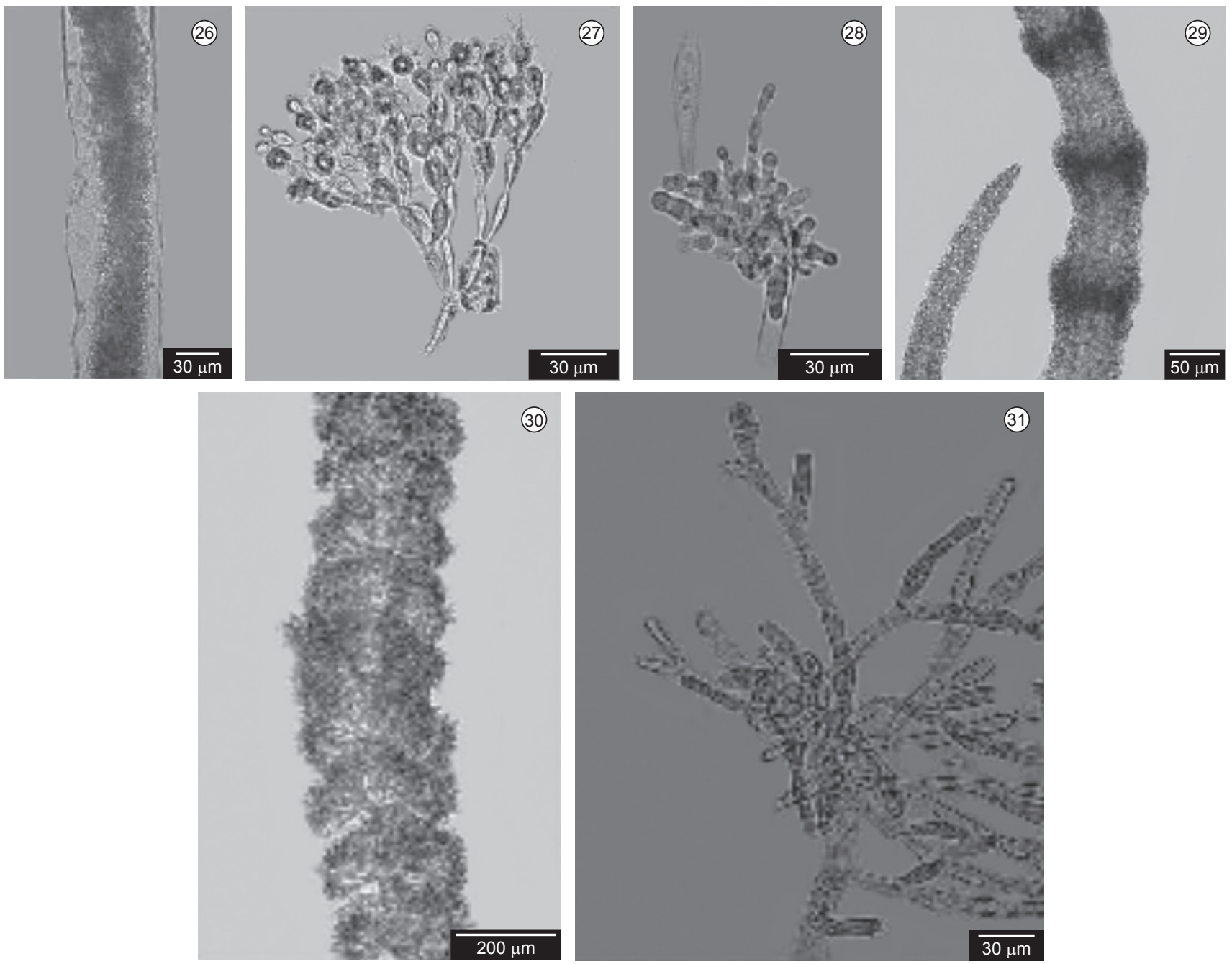

Figuras 26-32. 26) Vaucheria sp.; 27-28) Batrachospermum vogesiacum, 27) ramo primário, 28) carpogônio; 29) Batrachospermum puiggarianum; 30-31) Sirodotia delicatula, 30) vista geral dos verticilos, 31) carpogônio.

Figures 26-32. 26) Vaucheria sp.; 27-28) Batrachospermum vogesiacum, 27) primary fascicle, 28) carpogonium; 29) Batrachospermum puiggarianum; 30-31) Sirodotia delicatula, 30) general view of whorls, 31) carpogonium. 
indistinto; verticilos, densos ou laxos; obcônicos a obpiriformes, contíguos ou separados; internó 350-890 $\mu \mathrm{m}$ comprimento; células pericentrais ovóides ou cônicas, 2-3 fascículos primários; filamentos rizoidais pouco ou bem desenvolvidos; fascículos primários retos, 4-19 células; fascículos secundários numerosos, alongados; espermatângios esféricos ou obovóides, terminais ou subterminais, 5-9 $\mu \mathrm{m}$ diâmetro; ramo que sustenta o carpogônio reto ou levemente curvado, 1-6 células; carpogônio assimétrico, geralmente com uma protuberância semiglobular, 25-76 $\mu \mathrm{m}$ comprimento; tricogínio cilíndrico, alongado-cônico, elíptico, séssil ou pedicelado; carposporófito difuso e de forma não definida; filamentos gonimoblásticos eretos e prostrados desenvolvendo-se sobre o eixo principal.

Distribuição e características do riacho $(\mathrm{N}=1)$ : ponto 11 ; temperatura $18,2{ }^{\circ} \mathrm{C}$, condutividade específica $51,7 \mu \mathrm{S} . \mathrm{cm}^{-1}, \mathrm{pH} 7,1$, saturação de oxigênio $69 \%$, velocidade da correnteza $16 \mathrm{~cm} / \mathrm{s}$.

Comentários: Além da presença do gametófito desta espécie, também foi registrada a ocorrência do estágio "Chantransia". Este é o primeiro registro desta espécie para o estado do Paraná.

\section{Discussão}

Vinte e quatro macroalgas foram identificadas no presente estudo, incluindo 18 espécies e seis grupos vegetativos. Em adição, foi registrada também a presença do estágio "Chantransia" de espécies dos gêneros de algas vermelhas Batrachospermum e Sirodotia. O número global de espécies encontrado na região centro-oeste do estado do Paraná foi consideravelmente menor do que aqueles descritos para estudos similares (Sheath et al. 1989 - 67 táxons, Branco \& Necchi 1996 - 41), incluíndo regiões de clima extremo (Sheath et al. 1986 40) ou, então, regiões muito impactadas (Necchi et al. 1995 - 48)

A divisão algal predominante, em termos de número de táxons infra-genéricos, foi Chlorophyta com 13 espécies (54\%), seguida de Cyanophyta (5 espécies, 21\%), Rhodophyta e Heterokontophyta (3 espécies cada, 12,5\%). Os predomínios de algas verdes e azuis observados na área de estudos, basicamente, concordam com inúmeros trabalhos anteriormente desenvolvidos tanto para regiões tropicais quanto temperadas (Entwisle 1990 - 57\% Chlorophyta, $18 \%$ Cyanophyta, $14 \%$ Rhodophyta, $11 \%$ Heterokontophyta, Sheath et al. 1989 - 43\% Chlorophyta, 21\% Cyanophyta, 16\% Rhodophyta, 19\% Heterokontophyta, Sheath \& Cole 1992 - 35\% Chlorophyta, 24\% Cyanophyta, 20\% Rhodophyta, 21\% Heterokontophyta, Branco \& Necchi 1996 - 28,2\% Chlorophyta, 28,2\% Cyanophyta, 23,1\% Rhodophyta, 20,5\% Heterokontophyta, Necchi et al. 2000 40,6\% Chlorophyta, 32,6\% Cyanophyta, 14,9\% Rhodophyta, 11,9\% Heterokontophyta).

A espécie mais bem distribuída foi Phormidium retzii (C.Agardh) Gomont (Cyanophyta), ocorrendo em 29\% dos pontos de amostragem. Esta espécie tem sido reportada como cosmopolita e de ampla distribuição em vários estudos em ecossistemas lóticos. Sheath \& Cole (1992), em um estudo que amostrou 1.000 rios/riachos na América do Norte, encontraram $P$. retzii como a espécie de macroalga mais bem distribuída no continente. Necchi et al. (2000) também registraram esta espécie como predominante em riachos do Estado de São Paulo, assim como Branco et al. (2008) e Krupek et al. (2008) a reportaram como predominante em outras regiões do Estado do Paraná.

A proporção de tipos morfológicos foram as seguintes: emaranhados de filamentos (39\%), filamentos livres (26\%), tufos (18\%), colônias gelatinosas (9\%), filamentos gelatinosos (4\%) e crostas (4\%). Estas proporções estão de acordo com os dados reportados em estudos prévios (Necchi et al. 1995 - emaranhados de filamentos $31 \%$, filamentos livres $40 \%$, tufos $12,5 \%$, colônias gelatinosas $10,5 \%$, filamentos gelatinosos 6\%, Branco \& Necchi 1996 - emaranhados de filamentos $34 \%$, filamentos livres $22 \%$, tufos $15 \%$, colônias gelatinosas $7,5 \%$, filamentos gelatinosos $12 \%$, crostas 7,5\%, pseudoparenquimatoso $2 \%$, Peres et al. (2008) - emaranhados de filamentos $42,1 \%$, filamentos livres $21 \%$, tufos 5,3\%, colônias gelatinosas $15,8 \%$, filamentos gelatinosos 5,3\%, crostas 10,5\%). Os tipos morfológicos predominantes observados na área de estudos são considerados como bem adaptados ao estresse mecânico provocado pela velocidade da correnteza (Sheath \& Hambrook 1990), característica típica dos ambientes lóticos.

Todos os táxons já foram previamente reportados para ambientes lóticos brasileiros, porém cinco espécies representam novos registros para o Estado do Paraná, quais sejam: Tolypothrix distorta (Cyanophyta), Chaetophora pisiformis (Chlorophyta), Stigeoclonium fasciculare (Chlorophyta), Klebsormidium fluitans (Chlorophyta) e Sirodotia delicatula (Rhodophyta). Embora as amostragens não tenham produzido aumento no número de espécies descritas para a flora de macroalgas de riachos do Brasil, os registros para a região centro-oeste do Estado do Paraná são relevantes, uma vez que permitem ampliar o conhecimento sobre as distribuições geográfica e ambiental destes organismos no território nacional. Assim, estas novas informações podem fornecer subsídios para abordagens de biodiversidade, bem como autoecológicas que visem avaliar os limites de ocorrência ambiental destes táxons.

\section{Agradecimentos}

Este estudo contou com o apoio financeiro do CNPq, através do auxílio integrado no. 520257/01-4.

\section{Referências Bibliográficas}

ANAGNOSTIDIS, K. \& KOMÁREK, J. 1988. Modern approach to the classification system of cyanophytes. 3-Oscillatoriales. Algol. Stud. 50-53:327-472.

ANAGNOSTIDIS, K. \& KOMÁREK, J. 1990. Modern approach to the classification system of cyanophytes. 5-Stigonematales. Algol. Stud. 59:1-73.

BIGGS, B.J.F. \& PRICE, G.M.A. 1987. A survey of filamentous algal proliferation in New Zealand rivers. New. Zeal. J. Mar. Freshw. Res. 21:175-191.

BRANCO, C.C.Z., EMED, R.G., BRANCO, L.H.Z. \& NECCHI Jr., O. 2008 a. Macroalgas de riachos da Floresta Nacional de Irati, estado do Paraná, Sul do Brasil. Acta Bot. Bras. 22:433-447.

BRANCO, C.C.Z., KRUPEK, R.A. \& PERES, C.K. 2009. Ecological distribution of stream macroalgal communities from the mid-western region of Paraná State: evidence of the importance of local scale variation. Braz. Arch. Biol. Technol. No prelo.

BRANCO, C.C.Z. \& NECCHI Jr., O. 1996. Survey of stream macroalgae of eastern Atlantic Rainforest of São Paulo State, southeastern Brazil. Algol. Stud. 80:35-57.

BRANCO, L.H.Z., NECCHI Jr., O. 1997. Seasonality of macroalgae in three tropical drainage basins in São Paulo State, southeastern Brazil. Arch. Hydrobiol. 141:75-91.

BRANCO, L.H.Z. \& NECCHI Jr., O. 1998. Distribution of macroalgae in three tropical drainage basins of southeastern Brazil. Arch. Hydrobiol. 142:241-256.

BRANCO, L.H.Z., NECCHI Jr., O. \& BRANCO, C.C.Z. 1999. Cyanophyta from lotic ecosystems of São Paulo State, Southeastern Brazil. Algol. Stud. 94:63-87.

BRANCO, L.H.Z. \& PEREIRA, J.L. 2002. Evaluation of macroalgal communities along a gradient of organic pollution in a tropical stream. Arch. Hydrobiol. 155:147-161.

ENTWISLE, T.J. 1990. Macroalgae in Yarra River basin: flora and distribution. Proc. Royal Soc. 101:1-76. 
FILKIN, N.R., SHERWOOD, A.R. \& VIS, M.L. 2003. Macroalgae from 23 streams in the Hawaiian Islands. Pac. Sci. 57:421-431.

HOEK, C. Van den, MANN, D.G. \& JAHNS, H.M. 1995. Algae: an introduction to phycology. Cambridge University Press, Cambridge.

JOHN, D.M. \& MOORE, J.A. 1985. Observation on phytobenthos of freshwater Thames I. The environment, floristic composition and distribution of macrophytes (principally macroalgae). Arch. Hydrobiol. 102:435-459.

JOHANSSON, C. 1982. Attached algal vegetation in running waters of Jämtland, Sweden. Acta Phytogeogr. Suec. 71:1-83.

KAWECKA, B. 1980. Sessile alge in European mountain stream. 1: the ecological characteristic of communities. Acta Hydrobiol. 22:361-420.

KAWECKA, B. 1982. Stream ecossystem in mountain grassland (West Carpanthians). 6: Sessile algae communities. Acta Hydrobiol. 24:357-365.

KOMÁREK, J. \& ANAGNOSTIDIS, K. 1989. Modern approach to the classification system of cyanophytes. 4. Nostocales. Algol. Stud. $56: 247-345$.

KRUPEK, R.A., BRANCO, C.C.Z. \& PERES, C.K. 2008. Macroalgas de riachos da bacia do rio das Pedras, região Sul do Brasil. Hoehnea. 35:25-44.

LANDUCCI, M. \& LUDWIG, T.A.V. 2005. Diatomáceas de rios da bacia hidrográfica Litorânea, Paraná, Brasil: Coscinodiscophyceae e Fragilariophyceae. Acta Bot. Bras. 19:345-357.

LOWE, R. L. \& LaLIBERTE, G.D. 2006. Benthic Stream Algae: Distribution and Structure. In Methods in Stream Ecology (G. Lamberti \& F.R. Hauer, eds). Academic Press, Amsterdam, p. 327-356.

MAACK, R. 1981. Geografia física do Estado do Paraná. Livraria José Olympio Ed., Rio de Janeiro.

NECCHI Jr., O., BRANCO, C.C.Z., SIMÕES, R.C.G. \& BRANCO, L.H.Z. 1995. Distribution of stream macroalgae in northwest region of São Paulo State, southeastern Brazil. Hydrobiologia. 299:219-230.

NECCHI Jr., O., BRANCO, C.C.Z. \& BRANCO, L.H.Z. 2000. Distribution of stream macroalgae in São Paulo State, southeastern Brazil. Algol. Stud. 97:43-57.

NECCHI Jr., O., BRANCO, L.H.Z. \& BRANCO, C.C.Z. 2003. Ecological distribution of stream macroalgal communities from a drainage basin in the Serra da Canastra National Park, Minas Gerais, Southeastern Brazil. Braz. J. Biol. 63:1-12.

NECCHI Jr., O. \& PASCOALOTO, D. 1993. Seasonal dynamics of macroalgal communities in the Preto River basin, São Paulo, southeastern Brazil. Arch. Hydrobiol. 129:231-252.

NECCHI Jr., O., PASCOALOTO, D., BRANCO, C.C.Z. \& BRANCO, L.H.Z 1997. Stream macroalgal flora from the northwest region of São Paulo State, Southeastern Brazil. Algol. Stud. 84:91-112.

NECCHI Jr., O., PASCOALOTO, D. \& BRANCO, L.H.Z. 1994. Distribution of macroalgae in a tropical river basin from southeastern Brazil. Arch. Hydrobiol. 129:459-471.

PERES, C.K., BRANCO, C.C.Z. \& KRUPEK, R.A. 2008. Macroalgas de riachos da Serra da Prata, leste do Paraná, Sul do Brasil. Acta Bot. Bras. 22:333-344.

SANT'ANNA, C.L. \& AZEVEDO, M.T.P. 1995. Scytonemataceae (Cyanophyceae) from the State of São Paulo, southern Brazil. Nova Hedw. 46:519-539.

SHEATH, R.G. \& BURKHOLDER, J. 1985. Characteristics of softwater stream in Rhode Island. II: composition and seasonal dynamics of macroalgae communities. Hydrobiologia. 128:109-118.

SHEATH, R.G. \& COLE, K.M. 1992. Biogeography of stream macroalgae in North America. J. Phycol. 28:448-460.

SHEATH, R.G. \& HAMBROOK, J.A. 1990. Freshwater ecology. In COLE, K.M \& SHEATH, R.G. (Eds.). Biology of red algae. Cambridge University Press, Cambridge, p. 423-453.

SHEATH, R.G., HAMILTON, P.B., HAMBROOK, J.A. \& COLE, K.M. 1989. Stream macroalgae of eastern boreal forest region of North America. Can. J. Bot. 67:3553-3562.

SHEATH, R.G., MORISON, M.O., KORCH, J.E., KACZMARCZYK, D. \& COLE, K.M. 1986. Distribution of stream macroalgae in south-central Alaska. Hydrobiologia. 135:259-269.

VERB, R.G. \& VIS, M.L. 2001. Macroalgal communities from a acid mine drainage impacted watershed. Aquat. Bot. 71:93-107.

WONS, I. 1982. Geografia do Paraná. Editora Ensino Renovado, Curitiba.

Recebido em 10/11/08

Versão Reformulada recebida em 18/02/09

Publicado em 05/05/09 